\title{
Efficacy of two different types of island flaps for the repair of diabetic foot ulcers on the heel
}

\author{
Kailong Zhou", Zhenhua Zhu", Zhicheng Zuo, Jiaju Zhao \\ Department of Hand and Foot Surgery, The Second Affiliated Hospital of Soochow University, Suzhou, China \\ Contributions: (I) Conception and design: Z Zhu, K Zhou; (II) Administrative support: K Zhou; (III) Provision of study materials or patients: Z Zhu, \\ J Zhao, Z Zuo; (IV) Collection and assembly of data: Z Zhu, J Zhao; (V) Data analysis and interpretation: J Zhao, Z Zuo, K Zhou; (VI) Manuscript \\ writing: All authors; (VII) Final approval of manuscript: All authors. \\ \#These authors contributed equally to this work. \\ Correspondence to: Kailong Zhou. Department of Hand and Foot Surgery, The Second Affiliated Hospital of Soochow University, Suzhou 215004, \\ China. Email: zhoukailong1982@126.com.
}

Background: Heel ulcer of diabetic foot (DF) is a difficulty in clinical repair. The current study aimed to investigate the clinical efficacy of the medial plantar island flap (MPIF) and the sural nerve nutritional artery island flap (SNNAIF) for the repair of chronic diabetic foot ulcers (DFU) on the heel.

Methods: Twelve patients with chronic DFU on the heel were admitted to our department from August 2018 to August 2020. Upon admission, ulcer debridement and bone cement filling were performed for 2-3 weeks to control infection. Digital subtraction angiography (DSA) or computed tomography angiography (CTA) of the lower limb was performed to assess vascular status. Then, 5 patients were repaired with MPIF and 7 patients with SNNAIF.

Results: The MPIF survived completely in 5 cases; SNNAIF was used in 7 cases, and 6 cases survived completely. Meanwhile, 1 patient who underwent SNNAIF presented with partial necrosis of the distal end of the flap. Then, it healed after debridement and dressing changes. All 12 flaps were followed up for 6-12 months. The flaps had a soft texture, and their shape was satisfactory. In 2 cases, SNNAIFs re-ruptured 8 months after surgery. However, they healed after dressing changes and weight-bearing reduction. During the 10-month follow-up, the sensory recovery of MPIF in 5 cases was satisfactory because the flap contained medial plantar cutaneous nerve. Meanwhile, 7 patients who underwent SNNAIF repair had poor sensory recovery. All patients had good dorsiflexion and plantarflexion of the ankle with satisfactory function.

Conclusions: Both the MPIF and SNNAIF flaps had a high survival rate and are feasible for DFU repair with good clinical outcomes. If DSA or CTA shows that the medial plantar artery is unobstructed and the heel wound is small, MPIF can retain sensory function and wear resistance. It is the first choice for repairing diabetic foot ulcers on the heel. If the heel wound are large or DSA or CTA shows that the posterior tibial artery is occluded and the peroneal artery is unobstructed, SNNAIF repair is safer.

Keywords: Diabetic foot ulcers (DFU); medial plantar island flap (MPIF); sural nerve nutritional artery island flap (SNNAIF); repair

Submitted Dec 23, 2021. Accepted for publication Feb 16, 2022.

doi: $10.21037 /$ atm-22-293

View this article at: https://dx.doi.org/10.21037/atm-22-293

\section{Introduction}

Diabetes is a common, chronic, neurovascular disease that is challenging to treat because patients can have many serious complications, including diabetic foot ulcers (DFU) $(1,2)$.
According to the global epidemiological surveys, up to $25 \%$ of patients with diabetes develop foot ulcers. Among them, 14-24\% undergo amputation (3). DFU often occur in weight-bearing areas of the foot. The heel is a common 
site of DFU which are characterized by wounds that reach the deep anatomical layers, a long disease course, and repair difficulties (4). Advancements in microscopic techniques have promoted the widespread clinical application of different perforator flaps for the treatment of DFU. Flap therapy significantly reduces the amputation rate and improves quality of life (5). In previous reports, single skin flap was used to repair diabetic foot ulcers $(6,7)$, However, there are few comparative studies between the two kinds of flaps. From August 2018 to August 2020, 12 patients with chronic DF heel ulcers were admitted to the Department of Hand and Foot Surgery of The Second Affiliated Hospital of Soochow University and underwent medial plantar island flap (MPIF) and sural nerve nutritional artery island flap (SNNAIF) repair. The patients had good clinical outcomes. MPIF is a flap that carries the medial plantar artery and the cutaneous branch of medial plantar nerve, and SNNAIF is a flap whose blood is supplied by the most distal perforating branch of peroneal artery and whose axis is the running line of sural nerve. To contribute to the treatment of DF heel ulcers, the clinical efficacy of the two therapies was analyzed. We present the following article in accordance with the STROBE reporting checklist (available at https:// atm.amegroups.com/article/view/10.21037/atm-22-293/rc).

\section{Methods}

\section{General information}

In total, 12 patients were included in this study. All procedures performed in this study involving human participants were in accordance with the Declaration of Helsinki (as revised in 2013). This study was approved by the Ethics Committee of The Second Affiliated Hospital of Soochow University (JD-HG-2022-02). Because of the retrospective nature of the research, the requirement for informed consent was waived. Among them, 8 were men and 4 women, and they were aged between 48 and 72 (mean $=62$ ) years. All patients had type 2 diabetes and a disease history ranging from 5 to 20 years. Moreover, the course of their heel ulcers ranged from 1 to 3 months. In addition, the wounds presented with exudates and necrotic materials. In 7 cases, the ulcers reached as deep as the calcaneus, resulting in complicated osteomyelitis, and the heel wound size was about $2 \mathrm{~cm} \times 2 \mathrm{~cm}$ to $7 \mathrm{~cm} \times 8 \mathrm{~cm}$. Upon admission, ulcer debridement and bone cement filling were performed for 2 to 3 weeks to control infection. In addition, lower limb DSA or CTA was performed to evaluate vascular status.
Then, 5 and 7 patients underwent MPIF and SNNAIF repair, respectively.

\section{Surgical process}

\section{Preoperative preparation}

To control blood glucose levels to approximately $<8 \mathrm{mmol} / \mathrm{L}$, patients first received medical treatment including blood glucose control and anti-inflammatory medications and nutritional supplementation. We then obtained secretion samples for culture and changed the wound dressing, and adjust antibiotic drugs according to drug sensitivity test. Subsequently, foot radiography and lower limb vascular CTA or DSA were performed to evaluate the osteomyelitis status of the heel and the patency of the anterior and posterior tibial and peroneal artery and their perforating branches in the lower limb. In stage I, debridement and dilation procedures were performed, and the bone cement was filled for 2 to 3 weeks according to the degree of infection. After bone cement removal, the wound did not present with odor and purulent secretions, and infection was satisfactorily controlled. Then, in stage II, flap repairs were performed.

\section{MPIF}

(I) Flap design: before surgery, the medial plantar artery shape and the location of the perforating branches were detected via Doppler ultrasonography combined with CTA or DSA. The flap axis line was defined as the straight line from $1 \mathrm{~cm}$ below the prominence of the malleolus medialis and between the first and second metatarsal heads. The flap was designed in the non-weight-bearing area of the medial plantar area behind the metatarsal heads on both sides of the axis line, and the flap size was slightly larger than the wound surface by about $0.5 \mathrm{~cm}^{2}$. (II) Flap excision: an arc-shaped incision was made along the axis below the posterior aspect of the malleolus medialis. Next, the skin and subcutaneous tissue were dissected. If the posterior tibial artery, vein, and nerve were identified, dissection was performed gradually along the neurovascular bundle until the beginning of the abductor pollicis was exposed. Otherwise, it will lead to necrosis of the flap and failure of the operation. Dissection was performed from the beginning of the abductor pollicis, and the abductor pollicis was opened to expose the neurovascular bundle of the medial plantar. The flap was incised from the distal end and sides of the designed flap using the two-sided meeting method. Subsequently, the flap was excised from the plantar fascia, and the distal end of 
the medial plantar artery was incised and ligated. After flap excision, the cutaneous branch of the medial plantar nerve entering the flap was separated from the superior bundle of the medial plantar nerve trunk along the medial plantar artery and nerve bundle to the required length which is the distance from the rotation point to the ulcer wound. The separation was based on a distal to proximal sequence until the desired flap and tip were completely separated. (III) Wound treatment: an open or concealed channel of the flap was used to cover the heel wound with tension-free sutures. After complete hemostasis of the donor site, the abductor pollicis was repaired, and the wound was closed. In addition, after partial removal of the metatarsal fascia, the donor flap site was grafted with the full-thickness skin grafting method.

\section{SNNAIF}

(I) Flap design: the point approximately 4 to $6 \mathrm{~cm}$ above the posterior aspect of the lateral malleolus was set as the perforating point. Preoperative CTA or DSA and Doppler ultrasonography was used to show that the flap rotation point was the perforating point. The line from the midpoint of the Achilles tendon and the lateral malleolus to the midpoint of the popliteal fossa was set as the axis line, and the flap was designed in an inverted water-droplet shape according to wound size. The distance from the rotation point to the most distal end of the flap was slightly greater than the distance from the rotation point to the most distal point of the wound. Moreover, the flap area was greater than the wound by approximately $1 \mathrm{~cm}^{2}$. (II) Flap excision: we first dissected along the anterolateral aspect of the flap tip to the deep fascia. Next, the peroneal artery septal perforating branches between the tendon of the peroneus brevis and longus and the musculi soleus were explored. The diameter of the perforating branch was approximately $1.2 \mathrm{~mm}$. After determining the perforating branches, the proximal arc of the flap was incised to reveal the sural nerve and the small saphenous vein. During this procedure, the position and axis of the flap could be adjusted according to the location of the sural nerve and the small saphenous vein, so as to ensure that the sural nerve is located on the central axis of the flap. Subsequently, the anterior and posterior sides of the flap were excised, and the flap was completely separated from the deep fascial layer. The fascial tip of the flap, with a width of approximately $3 \mathrm{~cm}$, and a part of the dermal tip were preserved while ensuring that the peroneal nerve was contained within the flap. A triangular rotating flap with a subdermal vascular network was excised along the distal end of the rotation point to reduce tension on the tip of the flap after rotation and to prevent cat ear deformity. (III) Wound treatment: the heel ulcer was covered via an open or concealed channel, and the donor area was repaired with direct sutures or skin grafting.

\section{Postoperative management}

Patients were advised to take bed rest for 1 week after surgery to prevent pressure on the flap. The flap was kept warm with a heating lamp. In addition, the affected limb was elevated to promote flap reflux and reduce postoperative flap swelling. Anti-inflammatory, anticoagulant and antispasmodic treatment is needed after operation, and attention should be paid to controlling blood glucose, detecting liver and kidney function and supplementing albumin, and the flap blood supply was closely monitored. Generally, the flap was at risk of swelling during the 24 to 72 hours after surgery. If swelling occurred, a few stitches could be removed to relieve it. Wound status, particularly the nature of the exudate, was closely observed, and change the dressing every day if necessary. The donor site dressing was removed 1 week after surgery to observe flap survival. The stitches were removed 3 weeks after surgery, and the patient was instructed to do functional exercise, including active and passive flexion and extension of the ankle joint.

\section{Results}

All MPIFs and 6 SNNAIFs survived completely after surgery. Meanwhile, 1 SNNAIF had partial necrosis at the distal end of the flap. However, it healed after debridement and dressing changes. In total, 5 of 12 flaps presented with wound ooze, and they eventually healed after approximately 1 month of dressing changes. All 12 flaps were followed up for 6 to 12 months, with a mean follow-up duration of 10 months. All flaps had a soft texture, and their shape was satisfactory. In 2 cases, SNNAIFs re-ruptured 8 months after surgery. However, they healed after dressing changes and weight-bearing reduction. During the 10-month follow-up, 5 patients who underwent MPIF repair had satisfactory sensory recovery because of carrying the medial plantar cutaneous nerves. However, 7 patients who underwent SNNAIF repair had poor sensory recovery. All patients had good activities of ankle dorsiflexion and plantar flexion. 

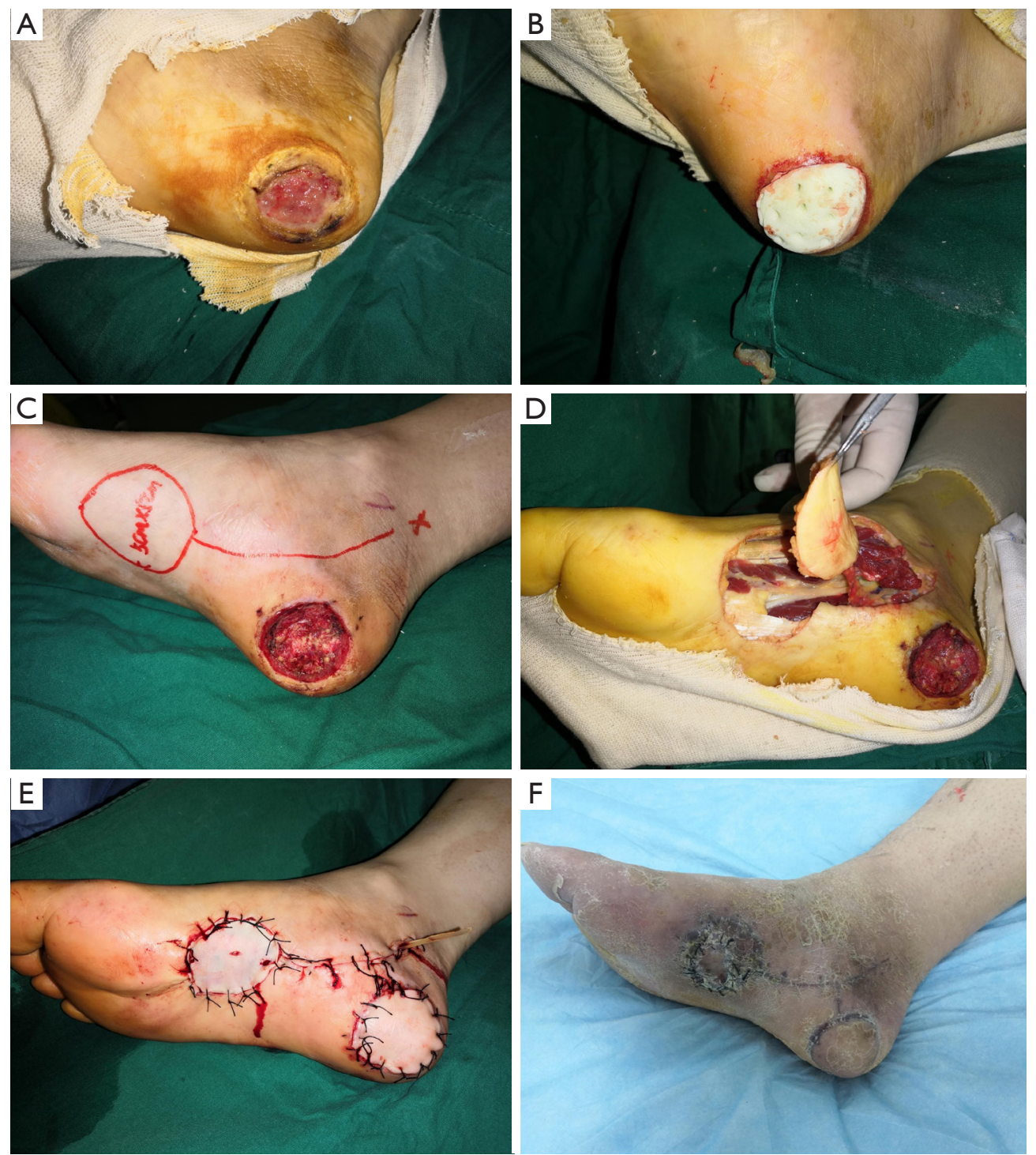

Figure 1 Case 1. (A) Diabetic foot ulcer in the right heel; (B) after ulcer debridement and bone cement filling; (C) design of the antegrade island flap on the medial side of the right plantar; (D) flap lifting during surgery; (E) appearance at the end of surgery; (F) postoperative appearance during the 10 -month follow-up.

\section{Typical cases}

\section{Case 1}

A 55-year-old male patient with a 17-year history of diabetes and poor glycemic control was admitted to The Second Affiliated Hospital of Soochow University because he had been experiencing soft tissue rupture and exudate on the right heel for more than 3 months. Physical examination revealed a chronic ulcer measuring approximately $4 \mathrm{~cm} \times 4 \mathrm{~cm}$ and a small volume of exudate on the right heel wound. Upon admission, the patient received aggressive glycemic control and anti-infection treatments. In stage I, the patient underwent debridement, dilation, and bone cement filling. In stage II, after wound debridement, the patient underwent MPIF repair. The flap healed completely after surgery. At the follow-up 10 months later, it had a soft texture, satisfactory function, and did not rupture (Figure 1).

\section{Case 2}

A 56-year-old female patient with a 12-year history of diabetes and poor glycemic control was admitted to 

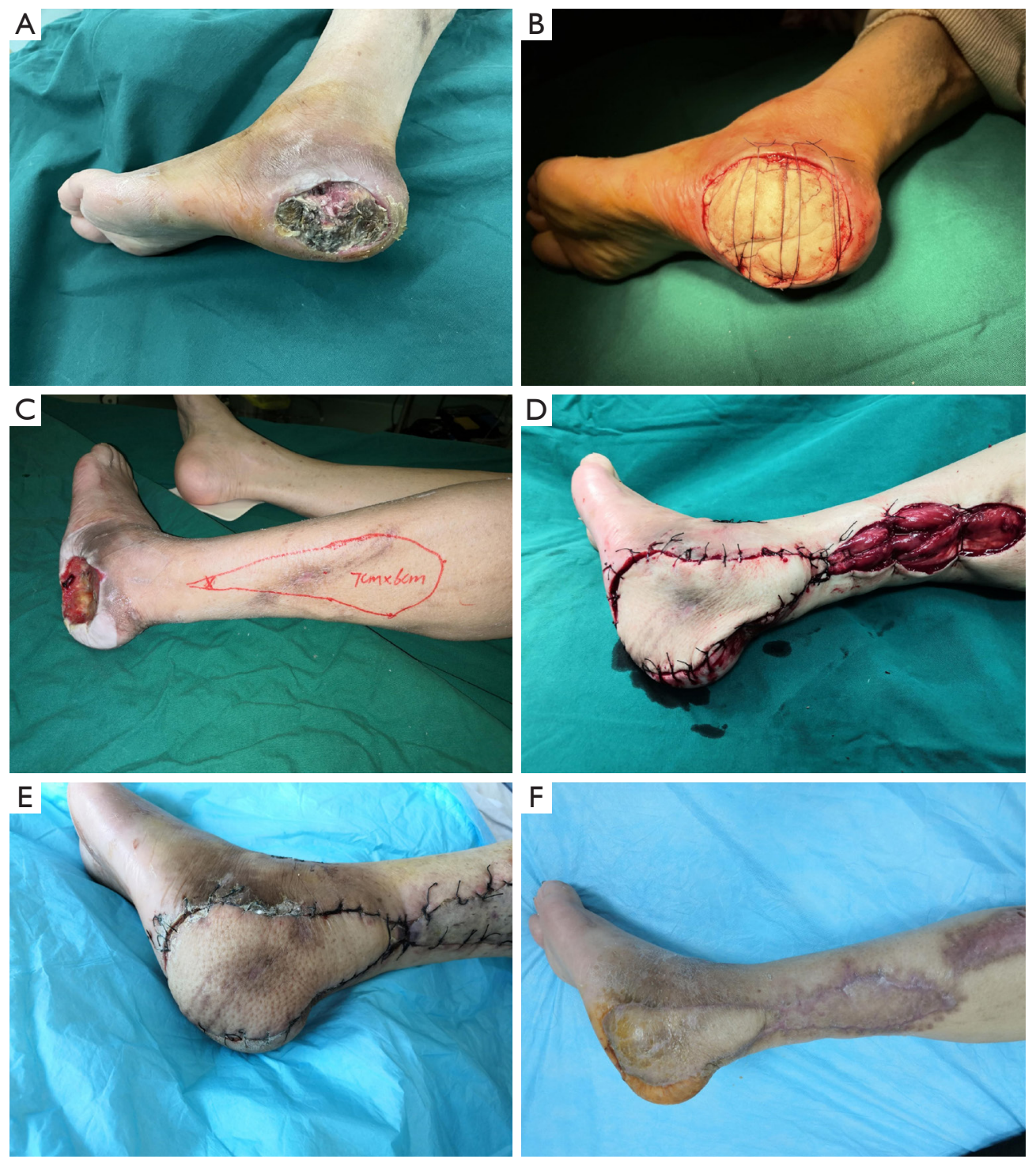

Figure 2 Case 2. (A) Diabetic foot ulcer in the left heel; (B) after ulcer debridement and bone cement filling; (C) design of the sural neurocutaneous island flap on the right leg; (D) flap covering the wound; (E) the flap survived completely 2 weeks after surgery; (F) postoperative appearance during the 10-month follow-up.

The Second Affiliated Hospital of Soochow University because she had been experiencing soft tissue rupture and exudate on the left heel for more than 2 months. Physical examination revealed a chronic ulcer measuring $6 \mathrm{~cm} \times 6 \mathrm{~cm}$, soft tissue skin necrosis, crusting, odor, and exudate on the left heel wound. Upon admission, the patient received aggressive glycemic control treatment. In stage I, the patient underwent debridement, dilation, and bone cement filling. In stage II, after wound debridement, the patient underwent SNNAIF repair. The flap healed completely after surgery. At the follow-up 10 months later, it had a soft texture, satisfactory function, and did not rupture (Figure 2).

\section{Discussion}

\section{Characteristics of DFU in the heel and its treatment peculiarities}

Diabetes is one of the four most threatening chronic diseases to human health, and DFU are among its serious 
complications and are a major cause of amputation $(8,9)$. Neuropathy, lower limb vasculopathy, and infection are the three main factors leading to the development of DFU (10). The heel is an area under high pressure and is therefore susceptible to pressure injury. Such an injury can lead to ulceration, and neuropathy in patients with diabetes can easily lead to sensory impairment in the heel. The capillaries in the heel are at risk of occlusion due to diabetic triggers, and the ulcer is more difficult to heal because of tissue ischemia. The thick cuticle and the loose tissues such as fat pads underneath the cuticle result in poor resistance to infection in the heel tissues. DF heel ulcers are common and persistent. If an ulcer has formed on the heel, it often leads to osteomyelitis on the calcaneus owing to its long course. In prolonged walking conditions, it can lead to calcaneal fractures and even amputation.

DF heel ulcers have certain peculiarities in terms of repair compared with ordinary chronic ulcers. First, due to vasculopathy among patients with diabetes, treatments for DF ulcers mainly include skin grafting and minor amputation to form a filleted flap for wound repair. However, as skin grafting is not wear-resistant and is at risk of rupture, and the wound of the heel cannot be repaired by a filleted flap because the calcaneus cannot be removed. Therefore, patients can only receive higher-risk therapies, such as island flap repair and free skin flap repair (11). In addition, due to poor peripheral blood supply to the foot in patients with DF, the trunk vessels of the lower leg or foot cannot be injured in ulcer repair. Carrying a trunk vessel will result in a higher flap survival rate. However, it will inevitably affect the blood supply to the foot and accelerate DF progression. Therefore, the treatment modality can only be based on the use of a perforator flap, with limited repair options and greater treatment difficulty. In view of the special characteristics of the heel skin and the weightbearing function of the heel, carrying the sensory nerves during flap repair of heel ulcers can prevent pressure damage to the greatest extent possible. Furthermore, as patients with diabetes cannot tolerate multiple procedures, flaps with a high survival rate should be selected to maximize success. Doing so can prevent the wound from growing and iatrogenic injury following a second surgery.

\section{Advantages and disadvantages of the two different types of island flaps for the repair of DF beel ulcers}

In terms of repair therapy for DF heel ulcers, the operative time should be kept as short as possible owing to the high number of comorbidities and the risk of anesthesia for patients undergoing surgery. With unobstructed vascular status, based on our clinical experience, the island flap is safer than the free skin flap, has a shorter operative time, and does not require strong microsurgical skills. Therefore, it is more applicable in primary hospitals. The island flap includes the prograde and retrograde forms. In our clinical practice, the flaps suitable for DF heel ulcer repair are MPIF (prograde form) and SNNAIF (retrograde form), each of which has its own advantages and disadvantages in clinical application. MPIF is mainly used to repair skin and soft tissue defects of heel and forefoot. It has been widely used in clinic. However, there are few reports about the repair of DF heel ulcers. The advantages of MPIF repair are as follows: first, the survival rate of flaps supplied with blood by the medial plantar artery is higher. Second, since the donor site is the medial non-weight-bearing area of the plantar foot, surgery does not affect the walking function of the foot. Third, as the blood to the forefoot is mainly supplied by the arteria dorsalis pedis and the lateral plantar artery, the flap pedicled with medial plantar artery had no effect on the blood supply of forefoot. Fourth, this type of repair allows the flap to carry the cutaneous branch of the medial plantar nerve to repair sensation in the heel. Therefore, it can be ideal for DF heel ulcer repair $(12,13)$. The disadvantages of the MPIF repair are as follows: first, due to the limited excision area (usually no more than $8 \mathrm{~cm} \times 4 \mathrm{~cm})(14)$, this type of repair therapy is restricted in larger ulcers. Second, the donor site requires skin grafting after excision, and there is a risk of skin graft necrosis. Third, DF is commonly accompanied by vascular diseases that are more likely to result in posterior tibial artery occlusion, which is a contraindication to MPIF repair.

In addition, the SNNAIF flap is most commonly used for the clinical repair of heel wounds $(15,16)$. Its blood is supplied by the anastomotic branches between the most distal septal perforating branch of the peroneal artery and the sural nerve nutrition blood vessel. The advantages of SNNAIF repair are as follows: first, the perforating branch is relatively constant. The flap is simple to excise and has a high survival rate. Moreover, it can be excised to a large extent (up to the upper middle of the calf) and can repair large heel wounds. Second, peroneal artery occlusion in the lower limb often occurs later than posterior and anterior tibial artery occlusion in patients with DF. Thus, SNNAIF has a reliable blood supply and is ideal for DF heel ulcer repair. Third, SNNAIF is a perforating flap, which does not damage the main blood vessels and can decrease the 
impact of blood supply to the DF. The disadvantages of SNNAIF repair are as follows: first, a SNNAIF may slip under external force and has poor wear resistance. Second, difficulty in anastomosing the cutaneous nerve with SNNAIF may result in poorer sensory recovery and the risk of recurrent rupture. Third, loss of lateral foot sensation after surgery may increase the risk of lateral foot skin rupture in patients with DFU.

\section{Selection of two different types of island flaps and relevant considerations}

These experiences can be used for reference in repairing DF heel ulcers with MPIF s and SNNAIFs. The choice of flap mainly depends on the patency of blood supply vessels of corresponding flap and the size of heel ulcer. First, preoperative preparation, including blood glucose control treatment and osteomyelitis classification (particularly based on the patency of the lower limb vessels), is important. If necessary, CTA or DSA can be performed to validate the patency of the anterior and posterior tibial vessels and peroneal artery. In patients with DF who present with uremia and who cannot undergo angiography, SNNAIF may be selected for wound repair as the peroneal artery may be the last vessel to be occluded, and MPIF is not recommended. Second, if the posterior tibial and peroneal arteries are patent, MPIF is recommended to repair small wounds in the heel because MPIF can carry the cutaneous branch of medial foot nerve, it can better restore the feeling of heel after operation. The flap excision site must be located in the arch area behind the weight-bearing area of the first metatarsal head to prevent affecting the weightbearing function of the foot after flap excision. Only the dermal nerve entering the flap should be retained to avoid damage to the sensory nerve bundle innervating the ventral part of the first and second toes of the foot. Due to the numerous vertical fibers in the heel, the subcutaneous tissue is less mobile, and in the process of transferring the flap, the subcutaneous tunnel may compress the neurovascular bundle. In this case, the skin can be cut to ensure the blood supply of the flap. In donor site repair, a part of the plantar fascia must be removed to ensure skin graft survival. Third, in cases where CTA or DSA indicates occlusion or severe stenosis of the posterior tibial vessels or a large heel wound, the MPIF area may exceed the non-weight-bearing area of the medial foot. Therefore, SNNAIF is an ideal option for repairing this type of wound. In addition, the septal perforating branch (which is located 4 to $6 \mathrm{~cm}$ above the posterior aspect of the lateral malleolus and has some variations) of the peroneal artery should be repositioned via Doppler ultrasonography before excision. The rotation point of the flap should be adjusted according to the perforating point to prevent damage to the perforating branch. Intraoperative ligation of the small saphenous vein with the distal tip prevents venous blood backflow and reduces postoperative flap swelling. At the distal end of the rotation point, a triangular subdermal vascular network can eliminate the cat ear deformity caused by island flap rotation.

In conclusion, the selection of an appropriate flap repair therapy is the prerequisite for effectively treating chronic DF heel ulcers. Due to poor vascular conditions among patients with diabetes, the available flap repair options are limited. According to current data, the MPIF and SNNAIF have a high survival rate. These two types of flaps have their own scopes of application and advantages and disadvantages. Therefore, a comprehensive assessment is required to select the best repair therapy for each patient. Our proposal is that if the medial plantar artery is unobstructed and the heel ulcer is small, MPIF is the first choice for the repair of diabetic foot heel ulcer. For the heel larger ulcer, posterior tibial artery occlusion, peroneal artery blood flow patency, SNNAIF covering the wound is safer. The deficiency of this study is that the sample size of clinical control study is too small. In the future, we need to further accumulate cases and improve the statistical analysis of postoperative data.

\section{Acknowledgments}

Funding: This research received financial support from the Program of Key Research and Development of Jiangsu Province (grant No. BE2018656), and the Key Medical Discipline in Suzhou (grant No. Szxk201802).

\section{Footnote}

Reporting Checklist: The authors have completed the STROBE reporting checklist. Available at https://atm. amegroups.com/article/view/10.21037/atm-22-293/rc

Data Sharing Statement: Available at https://atm.amegroups. com/article/view/10.21037/atm-22-293/dss

Conflicts of Interest: All authors have completed the ICMJE uniform disclosure form (available at https://atm. amegroups.com/article/view/10.21037/atm-22-293/coif). 
The authors have no conflicts of interest to declare.

Ethical Statement: The authors are accountable for all aspects of the work in ensuring that questions related to the accuracy or integrity of any part of the work are appropriately investigated and resolved. All procedures performed in this study involving human participants were in accordance with the Declaration of Helsinki (as revised in 2013). This study was approved by the Ethics Committee of The Second Affiliated Hospital of Soochow University (JDHG-2022-02). Because of the retrospective nature of the research, the requirement for informed consent was waived.

Open Access Statement: This is an Open Access article distributed in accordance with the Creative Commons Attribution-NonCommercial-NoDerivs 4.0 International License (CC BY-NC-ND 4.0), which permits the noncommercial replication and distribution of the article with the strict proviso that no changes or edits are made and the original work is properly cited (including links to both the formal publication through the relevant DOI and the license). See: https://creativecommons.org/licenses/by-nc-nd/4.0/.

\section{References}

1. Wang $\mathrm{H}, \mathrm{Xu} \mathrm{Z}$, Zhao M, et al. Advances of hydrogel dressings in diabetic wounds. Biomater Sci 2021;9:1530-46.

2. Dixon D, Edmonds M. Managing Diabetic Foot Ulcers: Pharmacotherapy for Wound Healing. Drugs 2021;81:29-56.

3. Jeffcoate WJ, Chipchase SY, Ince P, et al. Assessing the outcome of the management of diabetic foot ulcers using ulcer-related and person-related measures. Diabetes Care 2006;29:1784-7.

4. Kim HB, Altiparmak M, Pak CJ, et al. Reconstruction Using Free Flaps for Diabetic Heel Defects: Outcomes and Risk Factor Analysis. J Reconstr Microsurg 2020;36:494-500.

5. Kim K, Kim J, Jeong W, et al. Outcome of distal lower leg reconstruction with the propeller perforator flap in diabetic patients. J Plast Surg Hand Surg 2021;55:242-8.

6. Pallua N, Di Benedetto G, Berger A. Forefoot reconstruction by reversed island flaps in diabetic patients. Plast Reconstr Surg 2000;106:823-7.

7. Assi C, Samaha C, Chamoun Moussa M, et al. A Comparative Study of the Reverse Sural Fascio-Cutaneous
Flap Outcomes in the Management of Foot and Ankle Soft Tissue Defects in Diabetic and Trauma Patients. Foot Ankle Spec 2019;12:432-8.

8. Grant PJ, Cosentino F. The 2019 ESC Guidelines on diabetes, pre-diabetes, and cardiovascular diseases developed in collaboration with the EASD: New features and the 'Ten Commandments' of the 2019 Guidelines are discussed by Professor Peter J. Grant and Professor Francesco Cosentino, the Task Force chairmen. Eur Heart J 2019;40:3215-7.

9. Zhao H, McClure NS, Johnson JA, et al. A Longitudinal Study on the Association Between Diabetic Foot Disease and Health-Related Quality of Life in Adults With Type 2 Diabetes. Can J Diabetes 2020;44:280-286.e1.

10. Grennan D. Diabetic Foot Ulcers. JAMA 2019;321:114.

11. Yang $X$, Fang $Z$, Liu $M$, et al. Free vascularized fascia flap combined with skin grafting for deep toe ulcer in diabetic patients. J Surg Res 2018;231:167-72.

12. Scaglioni MF, Rittirsch D, Giovanoli P. Reconstruction of the Heel, Middle Foot Sole, and Plantar Forefoot with the Medial Plantar Artery Perforator Flap: Clinical Experience with 28 Cases. Plast Reconstr Surg 2018;141:200-8.

13. Liette MD, Ellabban MA, Rodriguez P, et al. Medial Plantar Artery Flap for Wound Coverage of the WeightBearing Surface of the Heel. Clin Podiatr Med Surg 2020;37:751-64.

14. Han Y, Han Y, Song B, et al. Free Medial Plantar Flap Versus Free Dorsal Myocutaneous Flap for the Reconstruction of Traumatic Foot Sole Defects. Ann Plast Surg 2020;84:S178-85.

15. Demiri E, Tsimponis A, Pavlidis L, et al. Reverse neurocutaneous vs propeller perforator flaps in diabetic foot reconstruction. Injury 2020;51 Suppl 4:S16-21.

16. Zhou L, Wei J, Liu L, et al. Composite sural neurocutaneous flap with gastrocnemius tendon for repairing defects of Achilles tendon and overlying soft tissue. J Orthop Surg (Hong Kong) 2020;28:2309499020971863.

(English Language Editor: C. Mullens)

Cite this article as: Zhou K, Zhu Z, Zuo Z, Zhao J. Efficacy of two different types of island flaps for the repair of diabetic foot ulcers on the heel. Ann Transl Med 2022;10(5):256. doi: 10.21037/atm-22-293 